\title{
New Properties over a New Type of Wreath Products on Monoids
}

\author{
Suha Ahmed Wazzan \\ Department of Mathematics, Science Faculty, King Abdulaziz University, Jeddah, Saudia Arabia \\ Email: swazzan@kau.edu.sa
}

How to cite this paper: Wazzan, S.A. (2019) New Properties over a New Type of Wreath Products on Monoids. Advances in Pure Mathematics, 9, 629-636. https://doi.org/10.4236/apm.2019.98032

Received: July 15, 2019

Accepted: August 27, 2019

Published: August 30, 2019

Copyright (C) 2019 by author(s) and Scientific Research Publishing Inc. This work is licensed under the Creative Commons Attribution International License (CC BY 4.0).

http://creativecommons.org/licenses/by/4.0/

\begin{abstract}
In [1], a new consequence of the (restricted) wreath product for arbitrary monoids $A$ and $B$ with an underlying set $A^{\oplus B} \times(A \times B) \times B^{\oplus A}$. Let us denote it by $A * B$. Actually, in the same reference, it has been also defined the generating and relator sets for $A * B$, and then proved some finite and infinite cases about it. In this paper, by considering the product $A * B$, we show Green's relations $\mathcal{L}$ and $\mathcal{R}$ as well as we present the conditions for this product to be left cancellative, orthodox and finally left (right) inverse(s).
\end{abstract}

\section{Keywords}

Wreath Product, Green's Relations, Left Cancellative Monoids, Orthodox Monoids, Left (Right) Inverse

\section{Introduction}

Let $A$ and $B$ be arbitrary monoids. In [2], Theorem 2.2, Howie and Ruskuc defined a presentation for the (restricted) wreath product of $A$ and $B$. Also, in [3], Theorem 7.1, it has been showed that the wreath product of semigroups satisfies the periodicity when these semigroups are periodic. In [1], a new derivation for wreath product of monoids $A$ and $B$ has been recently defined which will be dented by $A * B$ here (in [1], it has been denoted by $A \bowtie \triangleleft B$ but the author prefers her the symbol $*$ instead of $\triangleright \triangleleft$ to distinguish this new type of extension from the known symbol for general product (Zappa-Szép product $A \bowtie B)$ ). Also, again in [1], by proving the existence a presentation of this wreath product, it has been given necessary and sufficient conditions for $A * B$ to be regular and periodic, and some finite and infinite applications about it are denoted. In this paper, we give some algebraic properties of the new wreath product in terms of the algebraic properties of the monoids themselves. 
More specifically, we present the Green's relation $\mathcal{L}$ and $\mathcal{R}$ (in Section 2), and also prove the conditions on it to be left cancellative, orthodox and left (right) inverse.

We recall the fundamentals of the construction of $A * B$ which will be needed to form our results. We note that this product is based on the wreath product and we may refer to ([2] [4] [5] [6] [7]) for the details of wreath products. The Cartesian product of $B$ copies of the monoid $A$ is denoted by $A^{\times B}$, while the corresponding direct product is denoted by $A^{\oplus B}$, similar definition for $B^{\oplus A}$. One may think that $A^{\oplus B}$ and $B^{\oplus A}$ are the sets of all functions having finite support, that is to say, having the property that (b) $f=1_{A}$ for all but finitely many $b$ in $B$ and $(a) g=1_{B}$ for all but finitely many $a$ in $A$. The restricted wreath product of the monoid $A$ by the monoid $B$ is the set $A^{\oplus B} \times B$ with the multiplication defined by

$$
(f, b)\left(g, b^{\prime}\right)=\left(f^{b} g, b b^{\prime}\right),
$$

where, ${ }^{b} g: B \rightarrow A$ is given by

$$
(y)^{b} g=(y b) g \quad(y \in B) .
$$

Dually the restricted wreath product of the monoid $B$ by the monoid $A$ is the set $B^{\oplus A} \times A$ with the multiplication defined by

$$
(f, a)\left(g, a^{\prime}\right)=\left(f g^{a}, a a^{\prime}\right),
$$

where, $g^{a}: A \rightarrow B$ is given by

$$
(x) g^{a}=(a x) g \quad(x \in A) .
$$

Now for $P_{1}=\left(a_{1}, b_{1}\right), \quad P_{2}=\left(a_{2}, b_{2}\right) \in A \times B$, let us define

$$
P_{1} P_{2}=\left(a_{1} a_{2}, b_{1} b_{2}\right) \text {. }
$$

After that the new derivation for the wreath product of $A$ and $B$, denoted by $A * B$, is the set $A^{\oplus B} \times(A \times B) \times B^{\oplus A}$ with the multiplication

$$
\left(f, P_{1}, g\right)\left(h, P_{2}, k\right)=\left(f^{b_{1}} h, P_{1} P_{2}, g^{a_{2}} k\right) \text {, }
$$

where, ${ }^{b_{1}} h: B \rightarrow A$ and $g^{a_{2}}: A \rightarrow B$ are defined by

$$
(y)^{b_{1}} h=\left(y b_{1}\right) h \quad(y \in B)
$$

and

$$
(x) g^{a_{2}}=\left(a_{2} x\right) g \quad(x \in A) .
$$

In fact, $A * B$ is a monoid with the identity $\left(\overline{1},\left(1_{A}, 1_{B}\right), \tilde{1}\right)$, where $\overline{1}$ and $\tilde{1}$ are defined by

$$
\text { (b) } \overline{1}=1_{A}, \quad(a) \tilde{1}=1_{B},
$$

respectively, for all $b \in B$ and $a \in A$.

\section{Green's Relations on the Product $A * B$}

In the light of Green's relations, it is well known that one may prove some 
computational results (for example, the minimal number of generators etc.) on the monoid structure (which will be kept for a future work and so not investigated in here). Hence, in this section, we only characterize Green's relations $\mathcal{L}$ and $\mathcal{R}$ (cf. [8] [9]) for the product $A * B$.

Proposition 2.1 Let $A * B$ be the new derivation of wreath product of a monoid $A$ by a monoid $B$. Then

1) $\left(f_{1}, P_{1}, g_{1}\right) \mathcal{L}\left(h_{1}, P_{2}, k_{1}\right)$ in $A * B$ implies that $g_{1} \mathcal{L} k_{1}$ in $B^{\oplus A}$, and $P_{1} \mathcal{L} P_{2}$ in $A \times B$,

2) $\left(f_{1}, P_{1}, g_{1}\right) \mathcal{R}\left(h_{1}, P_{2}, k_{1}\right)$ in $A * B$ implies that $f_{1} \mathcal{R} h_{1}$ in $A^{\oplus B}$, and $P_{1} \mathcal{R} P_{2}$ in $A \times B$.

Proof. 1) Suppose that $\left(f_{1}, P_{1}, g_{1}\right) \mathcal{L}\left(h_{1}, P_{2}, k_{1}\right)$ in $A * B$. So there exist $\left(f_{2}, P_{3}, g_{2}\right),\left(h_{2}, P_{4}, k_{2}\right) \in A * B$ such that

$$
\begin{aligned}
& \left(f_{2}, P_{3}, g_{2}\right)\left(f_{1}, P_{1}, g_{1}\right)=\left(h_{1}, P_{2}, k_{1}\right), \\
& \left(h_{2}, P_{4}, k_{2}\right)\left(h_{1}, P_{2}, k_{1}\right)=\left(f_{1}, P_{1}, g_{1}\right) .
\end{aligned}
$$

These two equations can also be written as

$$
\begin{aligned}
& \left(f_{2}\left({ }^{b_{3}} f_{1}\right), P_{3} P_{1}, g_{2}^{a_{1}} g_{1}\right)=\left(h_{1}, P_{2}, k_{1}\right), \\
& \left(h_{2}\left({ }^{b_{4}} h_{1}\right), P_{4} P_{2}, k_{2}^{a_{2}} k_{1}\right)=\left(f_{1}, P_{1}, g_{1}\right) .
\end{aligned}
$$

Hence, by the equality of components, we obtain

$$
\begin{aligned}
& f_{2}\left({ }^{b_{3}} f_{1}\right)=h_{1}:: a_{3} a_{1}=a_{2},: b_{3} b_{1}=b_{2} \text { and } g_{2}^{a_{1}} g_{1}=k_{1}, \\
& h_{2}\left({ }^{b_{4}} h_{1}\right)=f_{1}:: a_{4} a_{2}=a_{1},: b_{4} b_{2}=b_{1} \text { and } k_{2}^{a_{2}} k_{1}=g_{1} .
\end{aligned}
$$

It follows that $g_{1} \mathcal{L} k_{1}$ in $B^{\oplus A}$ while $P_{1} \mathcal{L} P_{2}$ in $A \times B$.

Similar proof can be applied for 2). Hence the result.

Theorem 2.2 Assume that the product $A * B$ is obtained by a monoid $A$ and a group $B$. Then

$$
\left(f_{1}, P_{1}, g_{1}\right) \mathcal{R}\left(h_{1}, P_{2}, k_{1}\right) \in A * B \Leftrightarrow f_{1} \mathcal{R} h_{1} \in A^{\oplus B} \text { and } P_{1} \mathcal{R} P_{2} \in A \times B .
$$

Proof. By Proposition 2.1, $\left(f_{1}, P_{1}, g_{1}\right) \mathcal{R}\left(h_{1}, P_{2}, k_{1}\right) \in A * B$ implies the existence of $f_{1} \mathcal{R} h_{1} \in A^{\oplus B}$ and $P_{1} \mathcal{R} P_{2} \in A \times B$.

To prove the converse, let us suppose that $f_{1} \mathcal{R} h_{1}$ in $A^{\oplus B}$ and $P_{1} \mathcal{R} P_{2}$ in $A \times B$. In fact, $f_{1} \mathcal{R} h_{1}$ in $A^{\oplus B}$ gives that there exist $l_{1}$ and $l_{2}$ in $A^{\oplus B}$ such that

$$
f_{1} l_{1}=h_{1} \text { and } h_{1} l_{2}=f_{1} \text {. }
$$

Also, $P_{1} \mathcal{R} P_{2}$ in $A \times B$ implies that there exist $(c, d),\left(c^{\prime}, d^{\prime}\right)$ in $A \times B$ such that

$$
\left(a_{1}, b_{1}\right)(c, d)=\left(a_{2}, b_{2}\right) \text { and }\left(a_{2}, b_{2}\right)\left(c^{\prime}, d^{\prime}\right)=\left(a_{1}, b_{1}\right) .
$$

To show that $\left(f_{1}, P_{1}, g_{1}\right) \mathcal{R}\left(h_{1}, P_{2}, k_{1}\right)$ in $A * B$, we have to find two elements $\left(f_{2}, P_{3}, g_{2}\right)$ and $\left(h_{2}, P_{4}, k_{2}\right) \in A * B$ such that these must satisfy

$$
\left(f_{1}, P_{1}, g_{1}\right)\left(f_{2}, P_{3}, g_{2}\right)=\left(h_{1}, P_{2}, k_{1}\right)
$$




$$
\left(h_{1}, P_{2}, k_{1}\right)\left(h_{2}, P_{4}, k_{2}\right)=\left(f_{1}, P_{1}, g_{1}\right) .
$$

From these above, we obtain

$$
\begin{aligned}
& f_{1}\left({ }^{b_{1}} f_{1}\right)=h_{1}, P_{1} P_{3}=P_{2}, g_{1}^{a_{3}} g_{2}=k_{1}, \\
& h_{1}\left({ }^{b_{2}} h_{2}\right)=f_{1}, P_{2} P_{4}=P_{1}, k_{1}^{a_{4}} k_{2}=g_{1} .
\end{aligned}
$$

Since $A^{\oplus B}$ is a group (because $B$ is a group), we have

$$
\begin{aligned}
& g_{1}^{a_{3}} g_{2}=k_{1} \Rightarrow g_{2}=\left(g_{1}^{a_{3}}\right)^{-1} k_{1}, \\
& k_{1}^{a_{4}} k_{2}=g_{1} \Rightarrow k_{2}=\left(k_{1}^{a_{4}}\right)^{-1} g_{1} .
\end{aligned}
$$

Therefore, we set $f_{2}={ }^{\left(b_{1}\right)^{-1}} l_{1}$ and $\left.h_{2}={ }^{\left(b_{2}\right.}\right)^{-1} l_{2}$. Hence

$$
\begin{aligned}
\left(f_{1}, P_{1}, g_{1}\right)\left(f_{2}, P_{3}, g_{2}\right) & \left.=\left(f_{1}, P_{1}, g_{1}\right)\left({ }^{b_{1}}\right)^{-1} l_{1}, P_{3},\left(g_{1}^{a_{3}}\right)^{-1} k_{1}\right) \\
& =\left(f_{1}{ }^{b_{1}}\left({ }^{\left.b_{1}\right)^{-1}} l_{1}\right), P_{1} P_{3}, g_{1}^{a_{3}}\left(g_{1}^{a_{3}}\right)^{-1} k_{1}\right) \\
& =\left(f_{1}^{{ }_{1}} l_{1}, P_{1} P_{3}, k_{1}\right) \\
& =\left(h_{1}, P_{2}, k_{1}\right) .
\end{aligned}
$$

With a similar way, one can also show that

$$
\left(h_{1}, P_{2}, k_{1}\right)\left(h_{2}, P_{4}, k_{2}\right)=\left(f_{1}, P_{1}, g_{1}\right) \text {. }
$$

Hence, $\left(f_{1}, P_{1}, g_{1}\right) \mathcal{R}\left(h_{1}, P_{2}, k_{1}\right)$, as required.

\section{Some Algebraic Properties on $A * B$}

In this section, we will illustrate some algebraic properties of the new wreath product $A * B$ in terms of the algebraic properties of the monoids $A$ and $B$ themselves. The following Theorem characterize when new wreath product $M=A * B$ is a group.

Theorem 3.1 The new derivation of wreath product $M=A * B$ of monoids $A$ and $B$ is a group if and only if both $A$ and $B$ are groups.

Proof. Suppose $A$ and $B$ are both, groups, then $M=A * B$ is a monoid with identity $\left(\overline{1},\left(1_{A}, 1_{B}\right), \tilde{1}\right)$ where $\overline{1}$ and $\tilde{1}$ are defined by

$$
(b) \overline{1}=1_{A}, \quad(a) \tilde{1}=1_{B} .
$$

Now, let $(f,(a, b), g) \in M$. Define

$$
\begin{aligned}
& f^{\prime}: B \rightarrow A \text { by }(y) f^{\prime}=\left(\left(y b^{-1}\right) f\right)^{-1} \text { such that } f^{\prime} \in A^{\oplus B}, \text { and } y, b \in B, \\
& g^{\prime}: A \rightarrow B \text { by }(x) g^{\prime}=\left(\left(a^{-1} x\right) g\right)^{-1} \text { such that } g^{\prime} \in B^{\oplus A}, \text { and } x, a \in A .
\end{aligned}
$$

Then

$$
\begin{aligned}
(f,(a, b), g)\left(f^{\prime},\left(a^{-1}, b^{-1}\right), g^{\prime}\right) & =\left(f^{b} f^{\prime},\left(a a^{-1}, b b^{-1}\right), g^{a^{-1}} g^{\prime}\right) \\
& =\left(\overline{1},\left(1_{A}, 1_{B}\right), \tilde{1}\right)
\end{aligned}
$$


Since $(y) f^{b} f^{\prime}=(y) f(y b) f^{\prime}=(y) f\left(\left(y b b^{-1}\right) f\right)^{-1}=(y) f((y) f)^{-1}=1_{A}$, and $(x) g^{a^{-1}} g^{\prime}=(x) g^{a^{-1}}(x) g^{\prime}=\left(a^{-1} x\right) g\left(\left(a^{-1} x\right) g\right)^{-1}=1_{B}$. Hence $\left(f^{\prime},\left(a^{-1}, b^{-1}\right), g^{\prime}\right)$ is a right inverse for $(f,(a, b), g)$. Also

$$
\begin{aligned}
\left(f^{\prime},\left(a^{-1}, b^{-1}\right), g^{\prime}\right)(f,(a, b), g) & =\left(f^{\prime b} f,\left(a^{-1} a, b^{-1} b\right), g^{\prime a} g\right) \\
& =\left(\overline{1},\left(1_{A}, 1_{B}\right), \tilde{1}\right)
\end{aligned}
$$

Since $(y) f^{\prime b^{-1}} f=(y) f^{\prime}\left(y b^{-1}\right) f=\left(\left(y b^{-1}\right) f\right)^{-1}\left(y b^{-1}\right) f=1_{A}$, and $(x) g^{\prime a} g=(x) g^{\prime a}(x) g=(a x) g^{\prime}((x) g)^{-1}=\left(\left(a^{-1} a x\right) g\right)^{-1}(x) g=((x) g)^{-1}(x) g=1_{B}$. Hence, $\left(f^{\prime},\left(a^{-1}, b^{-1}\right), g^{\prime}\right)$ is a left inverse for $(f,(a, b), g)$, therefore, $M$ is a group.

Conversely, assume that $M=A * B$ is a group, let $\left(f,\left(1_{A}, 1_{B}\right), \tilde{1}\right)^{-1}=\left(k,\left(1_{A}, 1_{B}\right), l\right)$ so

$$
\left(f,\left(1_{A}, 1_{B}\right), \tilde{1}\right)\left(k,\left(1_{A}, 1_{B}\right), l\right)=\left(f^{1_{B}} k,\left(1_{A}, 1_{B}\right), \tilde{1}^{a} l\right)=\left(\overline{1},\left(1_{A}, 1_{B}\right), \tilde{1}\right)
$$

and

$$
\left(k,\left(1_{A}, 1_{B}\right), l\right)\left(f,\left(1_{A}, 1_{B}\right), \tilde{1}\right)=\left(k^{1_{B}} f,\left(1_{A}, 1_{B}\right), l^{a} \tilde{1}\right)=\left(\overline{1},\left(1_{A}, 1_{B}\right), \tilde{1}\right)
$$

Then $f^{1_{B}} k=\overline{1}, \quad \tilde{1}^{a} l=\tilde{1}, k^{1_{B}} f=\overline{1}$, and $l^{a} \tilde{1}=\tilde{1}$. Since

$$
\begin{gathered}
(b) f^{1_{B}} k=(b) \overline{1} \Rightarrow(b) f(b)^{1_{B}} k=1_{A} \Rightarrow(b) f\left(b 1_{B}\right) k=1_{A} \Rightarrow(b) f k=1_{A} \text { and } \\
(b) k^{1_{B}} f=(b) \overline{1} \Rightarrow(b) k(b)^{1_{B}} f=(b) \overline{1} \Rightarrow(b) k\left(b 1_{B}\right) f=1_{A} \Rightarrow(b) k f=1_{A} .
\end{gathered}
$$

Hence, $k=f^{-1} \in A^{\oplus B}$, therefore, $A^{\oplus B}$ is a group and hence $B$ is a group. Similarly we get $l=g^{-1} \in B^{\oplus A}$, if we suppose that $\left(\overline{1},\left(1_{A}, 1_{B}\right), g\right)^{-1}=\left(k,\left(1_{A}, 1_{B}\right), l\right)$, therefore $B^{\oplus A}$ is a group and hence $A$ is a group.

We first recall that a semigroup $S$ is called left-cancellative if $c a=c b \Rightarrow a=b$ and right-cancellative if $a c=b c \Rightarrow a=b$, for all $a, b, c \in S$ (cf. [8]). A semigroup is cancellative if it is left-cancellative and right-cancellative.

Theorem 3.2 $A$ and $B$ are cancellative monoids if and only if $M=A * B$ is cancellative monoid.

Proof. Assume that $A$ and $B$ are left cancellative monoids. Suppose

$$
\left(f, P_{1}, g\right)\left(k, P_{2}, l\right)\left(h, P_{3}, j\right) \in A * B,
$$

where $f, k, h \in A^{\oplus B}, g, l, j \in B^{\oplus A}$ and $P_{1}, P_{2}, P_{3} \in A \times B$. Therefore

$$
\begin{aligned}
\left(f, P_{1}, g\right)\left(h, P_{3}, j\right) & =\left(k, P_{2}, l\right)\left(h, P_{3}, j\right) \\
& \Rightarrow\left(f^{b_{1}} h, P_{1} P_{3}, g^{a_{3}} j\right)=\left(k^{b_{2}} h, P_{2} P_{3}, l^{a_{3}} j\right) \\
& \Rightarrow f^{b_{1}} h=k^{b_{2}} h, P_{1} P_{3}=P_{2} P_{3}, g^{a_{3}} j=l^{a_{3}} j \\
& \Rightarrow(\forall b \in B)(b) f^{b_{1}} h=(b) k^{b_{2}} h \\
& \Rightarrow(\forall b \in B)(b) f\left(b b_{1}\right) h=(b) k\left(b b_{2}\right) h \wedge b_{1}=b_{2}
\end{aligned}
$$

[Since $B$ is left cancellative]

$$
\Rightarrow f=k \wedge b_{1}=b_{2} \text {. }
$$

Also 


$$
\begin{aligned}
P_{1} P_{3}= & P_{2} P_{3} \text { and } g^{a_{3}} j=l^{a_{3}} j \\
\Rightarrow & (\forall a \in A)(a) g^{a_{3}} j=(a) l^{a_{3}} j \\
\Rightarrow & (\forall a \in A)(a) g^{a_{3}}(a) j=(a) l^{a_{3}}(a) j \\
\Rightarrow & (\forall a \in A)\left(a_{3} a\right) g(a) j=\left(a_{3} a\right) l(a) j \\
& {[\text { Since } A \text { is left cancellative }] } \\
\Rightarrow & (\forall a \in A)(a) g=(a) l \\
& {[\text { Since } B \text { is left cancellative }] } \\
\Rightarrow & g=l \wedge a_{1}=a_{2} .
\end{aligned}
$$

As a result, $M=A * B$ is actually a right cancellative monoid. In fact, one may prove with a similar way for left cancellative. Hence $M=A * B$ is cancellative.

On the other hand, the converse part of the proof is clear.

Hence the result.

In [10], the question of orthodox wreath products of monoids has been explained. After that, in [11], it has been investigated the orthodox wreath products of semigroups without unity. In this part, we will give necessary and sufficient conditions for $A * B$ to be orthodox, where $A$ and $B$ are any monoids.

Recall that the semigroup $S$ is called orthodox if the set of idempotents $E(S)$ is a subsemigroup of $S$. An orthodox semigroup $S$ is left (respectively, right) inverse if $e g e=g e$ (respectively, ege $=e g$ ) for every $e, g \in E(S)$. For more details reader refer [12] [13]. From [[10], Lemma 3.1], $A^{\oplus B}$ is an orthodox or left or right inverse semigroup if and only if $A$ has the same property. In the reference [1], the necessary and sufficient conditions for the new derivation of wreath products to be regular have been defined. In here, we give sufficient conditions for it to be orthodox as in the following theorem.

Theorem 3.3 If $A * B$ is an orthodox monoid or left (right) inverse, then each of $A$ and $B$ has the same property.

Proof. Applying [[1], Theorem 5.2], we see that $A$ and $B$ are regular. It remains to prove that the set of idempotents of $A$ and $B$ closed under multiplication defined on $A$ and $B$ respectively. Let $f, g \in E\left(A^{\oplus B}\right)$ and $h, k \in E\left(B^{\oplus A}\right)$. Then $f^{2}=f, g^{2}=g, h^{2}=h$ and $k^{2}=k$. Since $A * B$ is an orthodox monoid, then for an element $(f,(a, b), h),(g,(c, d), k) \in E(A * B)$, we have

$$
\begin{aligned}
& (f,(a, b), h),(g,(c, d), k)=\left(f^{b} g,(a c, b d), h^{c} k\right) \in E(A * B) \\
\Rightarrow & \left(f^{b} g,(a c, b d), h^{c} k\right)\left(f^{b} g,(a c, b d), h^{c} k\right)=\left(f^{b} g,(a c, b d), h^{c} k\right) \\
\Rightarrow & \left(\left(f^{b} g\right)^{b d} f^{b} g,\left((a c)^{2},(b d)^{2}\right),\left(h^{c} k\right)^{a c}\left(h^{c} k\right)\right)=\left(f^{b} g,(a c, b d), h^{c} k\right) \\
\Rightarrow & \left(f^{b} g\right)^{b d} f^{b} g=f^{b} g,\left(h^{c} k\right)^{a c}\left(h^{c} k\right)=h^{c} k,(a c)^{2}=a c,(b d)^{2}=b d \\
\Rightarrow & (f g)^{2}=f g \text { in } A^{\oplus B} \text { and }(h k)^{2}=h k \text { in } B^{\oplus A} .
\end{aligned}
$$

Furthermore 


$$
\begin{aligned}
(x)\left(f^{b} g\right)^{b d} f^{b} g & =(x) f^{b} g \\
& \Leftrightarrow(b d x)\left(f^{b} g\right)(x) f(x)^{b} g=(x) f(x)^{b} g \\
& \Leftrightarrow(b d x) f(b d x)^{b} g(x) f(x b) g=(x) f(x b) g \\
& \Leftrightarrow(b d x) f(b d x b) g(x) f(x b) g=(x) f(x b) g \\
& \Leftrightarrow(f g)^{2}=f g \text { for every } x \in B .
\end{aligned}
$$

Similar, calculation shows that $(h k)^{2}=h k$, for every $y \in A$. Hence the set of idempotents of $A^{\oplus B}$ and $B^{\oplus A}$ are subsemigroups. The result follows from [[10], Lemma 3.1].

Now let us suppose that $A * B$ is the left inverse. Then, for any element $(f,(a, b), h),(g,(c, d), k) \in E(A * B)$, where $f, g \in E\left(A^{\oplus B}\right)$ and $h, k \in E\left(B^{\oplus A}\right)$, we certainly have

$$
\begin{aligned}
& (f,(a, b), h)(g,(c, d), k)(f,(a, b), h)=(g,(c, d), k)(f,(a, b), h) \\
\Rightarrow & \left(f^{b} g^{b d} f,(a c a, b d b), h^{c a} k^{a} h\right)=\left(g^{d} f,(c a, d b), k^{a} h\right) \\
\Rightarrow & f^{b} g^{b d} f=g^{d} f, h^{c a} k^{a} h=k^{a} h, a c a=c a, b d b=d b \\
\Rightarrow & f g f=g h, h k h=k h
\end{aligned}
$$

and

$$
\begin{aligned}
& (x) f^{b} g^{b d} f=(x) g^{d} f \\
\Leftrightarrow & (x) f(x)^{b} g(x)^{b d} f=(x) g(x)^{d} f \\
\Leftrightarrow & (x) f(x b) g(x b d) f=(x) g(x d) f .
\end{aligned}
$$

Hence, $f g f=g h$ for every $x \in B$. Similar, calculation shows that $h k h=k h$ for every $y \in A$. We thus conclude that $A$ and $B$ are actually left inverses.

The same proof can be applied to show right inverse case as well.

Note 3.4 1) The other inclusion of Theorem 3.3 is left for future work. Following Caito [10], who determined necessary and sufficient conditions for the (restricted) wreath product to be orthodox, to be left inverse and to be right inverse, respectively.

2) There is also a particular class of regular monoids, namely coregular monoids [14]. An element of a monoid $S$ is called coregular if there is a $\beta \in S$ such that $\alpha=\alpha \beta \alpha=\beta \alpha \beta$ as well as the monoid $S$ is called coregular if each element of it is coregular cf. [15]. In fact, the coregularity and its properties over the new type of wreath product are left as an open problem for the future studies.

\section{Conclusion}

In this paper, the author investigated some specific theories such as Green's relations, left cancellative, orthodox, left (right) inverse etc. over new type of wreath products over monoids. Of course, there are still so many different properties that can be checked on this important product. On the other hand, in Note 3.4, we indicated some problems for future studies. 


\section{Conflicts of Interest}

The authors declare no conflicts of interest regarding the publication of this paper.

\section{References}

[1] Wazzan, S.A., Cevik, A.S. and Ates, F. (2019) The New Derivation for Wreath Products of Monoids. Filomat.

[2] Howie, J.M. and Ruskuc, N. (1994) Construction and Presentations for Monoids. Communications in Algebra, 22, 6209-6224.

https://doi.org/10.1080/00927879408825184

[3] Robertson, E.F., Ruskuc, N. and Thomson, M.R. (2002) On Finite Generation and Other Finiteness Conditions for Wreath Products of Semigroups. Communications in Algebra, 30, 3851-3873. https://doi.org/10.1081/AGB-120005823

[4] Baumslag, G. (1961) Wreath Products and Finitely Presented Groups. Mathematische Zeitschrift, 75, 22-28. https://doi.org/10.1007/BF01211007

[5] Cevik, A.S. (2000) The Efficiency of Standard Wreath Product. Proceedings of the Edinburgh Mathematical Society, 43, 415-423. https://doi.org/10.1017/S0013091500021003

[6] Cevik, A.S. (2003) The p-Cockcroft Property of Semi-Direct Products of Monoids. International Journal of Algebra and Computation, 13, 1-16. https://doi.org/10.1142/S0218196703001298

[7] Meldrum, J.D.P. (1995) Wreath Products of Groups and Semigroups. Longman, Harlow.

[8] Howie, J.M. (1995) Fundamentals of Semigroups Theory. Clarendon Press, Oxford.

[9] Lawson, M.V. (1998) Inverse Semigroups: The Theory of Partial Symmetries. World Scientific, Singapore. https://doi.org/10.1142/3645

[10] Saito, T. (1989) Orthodox Semidirect Products and Wreath Products of Monoids. Semigroup Forum, 38, 347-354. https://doi.org/10.1007/BF02573242

[11] Zhang, R. (1999) A Note on Orthodox Semidirect Products and Wreath Products of Monoids. Semigroup Forum, 58, 262-266. https://doi.org/10.1007/s002339900019

[12] Hickey, J.B. and Lawson, M.V. (1997) Unit Regular Monoids. Proceedings of the Edinburgh Mathematical Society, 127A, 127-144. https://doi.org/10.1017/S0308210500023532

[13] McFadden, R.B. (1984) Unit-Regular Orthodox Semigroups. Glasgow Mathematical Journal, 25, 229-240. https://doi.org/10.1017/S0017089500005656

[14] Bijev, G. and Todorov, K. (1980) Coregular Semigroups. Notes on Semigroups VI, Budapest (1980-1984), 1-11.

[15] Dimitrova, I. and Koppitz, J. (2011) Coregular Semigroups of Full Transformations. Demonstratio Mathematica, 44, 739-753. https://doi.org/10.1515/dema-2013-0342 